\title{
Environmental Science Nano
}

Check for updates

Cite this: Environ. Sci.: Nano, 2019, 6,2520

Received 25th April 2019,

Accepted 24th June 2019

DOI: $10.1039 / c 9 e n 00472 f$

rsc.li/es-nano

\section{Prospective environmental risk assessment of nanocellulose for Europe $\dagger$}

\author{
Natasha Stoudmann, (D) Bernd Nowack (D) and Claudia Som (D) *
}

The many appealing properties of nanocellulose have led to increasing interest in the material from research and industry over the past years, with the material showing potential for both industrial and consumer goods. This will unavoidably lead to increasing release of nanocellulose into the environment. Although nanocellulose is largely regarded as non-toxic, knowledge gaps surrounding its impacts on the environment and human health still exist and data remains scarce. This study aimed to quantitatively assess the environmental risk of nanocellulose by characterizing both environmental exposure and hazard. Firstly, a probabilistic species sensitivity distribution (PSSD) was developed to assess the hazard by calculating the predicted no effect concentration (PNEC) of the surface water compartment, resulting in a PNEC of 7.8 $\mathrm{mg} \mathrm{l}^{-1}$ (mean value of the distribution). Secondly, the dynamic probabilistic material flow assessment (DPMFA) method was employed to assess the exposure by quantifying the predicted environmental concentration (PEC), using the European Union as a system boundary, and 2000 to 2025 as timeframe. This resulted in a PEC in surface water of $0.23 \mu \mathrm{g} \mathrm{l}^{-1}$ in 2015 and $2.37 \mu \mathrm{g} \mathrm{l}^{-1}$ in 2025 (mean values). The PEC and PNEC distributions allowed for the calculation of the risk characterization ratio (RCR). Results show an RCR of $6.9 \times 10^{-5}$ in 2015 , and $7.1 \times 10^{-4}$ in 2025 , implying that under the chosen assumptions there is no present or future environmental risk surrounding nanocellulose within the surface water compartment, even assuming a compound annual growth rate of $19 \%$ for nanocellulose production in upcoming years.

\section{Environmental significance}

Increasing nanomaterials in commercial products necessarily leads to increasing concentrations of these materials within the environment. Quantifying the risk this may pose at an early stage of material development is therefore useful. Nanocellulose is still in early stages of commercialization, but production volumes are expected to grow significantly in the upcoming years. This study aimed to quantify the environmental risk surrounding this novel material while accounting for expected future production growth. Results from this work contribute to the growing body of environmental risk assessments of nanomaterials, and open the way for the safe commercialization of nanocellulose-containing products.

\section{Introduction}

Nanocellulose (NC) has been gaining increasing attention from both research and industry over the past years. The material can be derived from a multitude of abundant cellulosic biomass sources such as wood pulp, agricultural crops, organic waste, as well as from bacteria. ${ }^{1}$ Its physico-chemical properties, including high tensile strength, biocompatibility, and high aspect ratio make it attractive to a wide range of sectors, spanning from medical to construction. ${ }^{2}$ As nanocellulose originates from renewable matter, its potential to replace petroleum-derived materials for example for films, coat-

Empa - Swiss Federal Laboratories for Materials Science and Technology, Technology and Society Laboratory, Lerchenfeldstrasse 5, CH-9014 St. Gallen, Switzerland.E-mail: claudia.som@empa.ch

$\dagger$ Electronic supplementary information (ESI) available. See DOI: 10.1039/ c9en00472f ings, composites, and packaging are particularly interesting in the wake of current political and societal movements towards reduction of plastic consumption. ${ }^{3-5}$

Three categories of NC exist: cellulose nanocrystals (CNC), cellulose nanofibers (CNF), and bacterial nanocellulose (BNC). The possibility of controlling its biofabrication make BNC particularly appealing to the biomedical industry. ${ }^{6}$ In addition to being produced in different ways, these three types also vary in their physico-chemical properties, from size to crystallinity. ${ }^{7}$ Most of the NC being developed for commercial purposes is in the form of $\mathrm{CNF}$, with CNC patents largely being owned by universities and public institutions. ${ }^{8,9}$ Currently, many NC-based applications are at the pilot scale or going through industry trials, with a first few applications having already entered the market. ${ }^{10}$ It is expected that many applications will reach commercialization within the next 510 years. ${ }^{11}$ Forecasts regarding NC production volumes vary 
substantially, but are overall optimistic. ${ }^{12}$ The anticipated increase in NC production and use will unavoidably lead to increased release into the environment and to increased human exposure to the material at various product life stages.

At the nanoscale, the behavior and physicochemical properties of materials change, giving them new qualities, but also having the potential to negatively impact human health and the environment. ${ }^{13-16}$ Although NC is largely considered non-toxic, as is its bulk form, ecotoxicity studies remain scarce and knowledge gaps remain. A number of reviews surrounding the material's characterization and toxicity have been published in recent years. ${ }^{15,17-19}$ Their overarching conclusions are that uncertainties are still present, preventing a straightforward judgement to be made regarding health and safety issues surrounding the material. Shatkin and $\mathrm{Kim}^{20}$ undertook a life cycle risk assessment of NC by identifying exposure scenarios and evaluating its toxicity. The resulting health and safety roadmap allows for a qualitative overview of the material, with the authors highlighting the existence of remaining data gaps and research needs. Endes et al. ${ }^{15}$ reviewed the current knowledge of the impacts of NC on human and environmental health. The authors identified wide divergences between study findings, and pointed out the current lack of chronic, low dose, and repeated exposure studies.

There are currently no methods allowing to directly measure concentrations of engineered nanomaterials (ENMs) in the environment. Using a modelling approach is therefore necessary. ${ }^{21}$ Such a top-down procedure allows environmental concentrations to be estimated based on demand volumes and other input data. ${ }^{22-24}$ This approach has been used to assess the environmental risk of a number of ENMs, including nano-SiO ${ }_{2}$, nano-Ag, nano- $\mathrm{TiO}_{2}$, nano-ZnO, carbon nanotubes (CNTs), fullerenes, nano-Au, and nano- $\mathrm{CeO}_{2} \cdot{ }^{25-29}$ Our study follows a similar structure as that of Wang et al. ${ }^{28}$ who looked at the environmental release and risks of nano- $\mathrm{SiO}_{2}$ through the use of probabilistic material flow and environmental risk assessment models. By using such an approach, this study aims to contribute towards filling some of the remaining gaps surrounding $\mathrm{NC}$ by quantitatively assessing the environmental risk of the material. Due to limited information and data availability, we consider all forms of NC, without differentiating between $\mathrm{CNC}, \mathrm{CNF}$, and BNC.

\section{Methodology}

\subsection{Exposure assessment}

Exposure was characterized by modelling the stocks and flows of NC throughout its life cycle, which includes production, manufacturing, use, and end-of-life (EoL), using a dynamic probabilistic material flow analysis (DPMFA). ${ }^{30}$ Our system boundary was the European Union, and the timeperiod considered from 2000 to 2025 . We used the model described in Sun et al. ${ }^{31}$ as a base model, which we configured to fit the system and data under consideration. The dynamic aspect of the MFA was incorporated in order to account for the historical inputs and releases of NC, thereby modeling changes of the system over time. ${ }^{30,32}$ Furthermore, it allows for a prospective assessment by evaluating how the system is predicted to develop in the future.

The probabilistic nature of the MFA allows the uncertainties surrounding input parameters (demand volume, allocation to product categories and transfer coefficients) to be taken into account by treating these inputs as probability distributions. Two types of distributions were applied to the various data sources, namely triangular and trapezoidal, as explained in detail in Sun et al. ${ }^{33}$ Following the studies by Sun et al. and Gottschalk et al., ${ }^{33,34}$ we used two degree of belief (DoB) values to evaluate the reliability of data. These were $80 \%$ for high reliability data, from peer-reviewed studies and market reports, and $20 \%$ for data from presentations, reports, and other sources giving little methodological detail. These DoB translated into different sized samples of the corresponding parameter in the Monte Carlo simulation, used to account for the uncertainties through a stochastic approach. $^{33,35}$

The model is made up of various technical and environmental compartments, from and into which NC flows. The four environmental compartments of the model are 'air', 'surface water', 'subsurface water' and 'soil' (divided into 'natural and urban soils' and 'sludge treated soils'). The seven technical compartments are 'production', 'manufacturing', and 'consumption' ('PCM'), 'sewage treatment plants' ('STP'), 'waste incineration plants' ('WIP'), 'landfills', 'reprocessing', 'elimination', and 'export'. Within our model, 'export' represents export of nanocellulose-containing waste from Europe. We do not consider product imports or exports, as we assume that the use phase of all products takes place within the system boundary. The 'reprocessing' compartment represents recycling and further conversion of the products and material within the system, and 'elimination' represents the destruction and loss of the material through combustion while in the WIP.

Three sources were used as NC production volume input in the model. These varied significantly in terms of forecasted volumes, but as there is high uncertainty regarding current and future production, all three were considered, with various DoBs being attributed to each. Values from Future Markets $^{9}$ were given a DoB of $80 \%$. The two sources from the report by Miller, ${ }^{36}$ which presents NC market forecasts from various companies and institutes, were given a DoB of $20 \%$, as the report does not detail how these volumes were reached. We only used the forecasts in the report by Miller $^{36}$ which specified a time horizon, not just potential volume. When the volume of only a single year was given, we extrapolated past and future volumes by assuming a compound annual growth rate (CAGR) of 19\% between 2015 and 2025. ${ }^{37}$ We did not use the forecast made by RISI for $2025,{ }^{12}$ as the estimated 450000 tons per year of global production was deemed unrealistic by expert judgement. Although the study by Cowie et $a l^{38}$ estimates global and United States NC production volumes through market projections, their study 
was focused on volume potential, without considering a specific timeline or year in which these projected volumes might be reached. We therefore did not consider the volumes discussed in their study, although did consider the shares of NC attributed to various product categories that were identified. Furthermore, although data regarding the capacity of NC production plants were available in various studies, they were considered unreliable as estimations of actual production volumes, as these facilities are not currently producing to maximum capacity.

European volumes were scaled from global volumes based on Europe's current and projected NC market share. According to Future Markets, ${ }^{9}$ Europe currently represents $33 \%$ of the global NC market. This share is projected to increase to $34 \%$ by 2027 , and we assume that this would already be the case by 2025 . No information regarding past NC production volumes were found. We therefore assumed demand to be correlated with trends in patents and scientific publications. Accordingly, production was set to 0 tons in the year 2000 (earliest considered year in this study), following a linear growth between 2000 and $2015,{ }^{8,39}$ with a CAGR of $21.3 \%$.

How much and where an ENM is released will depend on the product in which it is incorporated and its matrix, rather than on the ENM itself. ${ }^{40-42}$ The attribution of nanocellulose to product categories is therefore a crucial step in modelling its later release. Nanocellulose is still in early stages of commercialization, with the majority produced going into research and development (R\&D). ${ }^{2,9}$ To account for this, we treated product category shares dynamically, as the share of nanocellulose going into $R \& D$ will slowly decrease relatively over time. All other product categories and their shares were developed based on a literature search of nanocellulose applications, where the maximal, minimal and mean shares were used to develop the distribution for each product categories. 9,36,38 Assuming a linear growth, by 2025, 58\% of produced nanocellulose will be used for R\&D purposes, down from $75 \%$ in 2015 . The coefficient of variation of the $R \& D$ product category was set to $50 \%$, to account for the high uncertainty surrounding this value. ${ }^{22}$

Information regarding release of nanocellulose to various environmental and technical compartments, required to determine transfer coefficients, was extremely limited. We therefore used transfer coefficients from studies that looked at the release of ENMs with similar properties and having similar product categories as those identified for NC, for example CNT and nano-SiO 2 (e.g.,ref. 29, 31 and 43). The same was the case for the release schedule from product categories, as no studies regarding the release of NC from products or NC-containing materials were identified in the literature.

Allocations of product categories to solid waste categories were based on the study by Adam and Nowack. ${ }^{44}$ These waste categories include mixed municipal solid waste (MMSW), packaging waste, waste electrical and electronic equipment (WEEE), textile waste, and construction and demolition waste (CDW). Four additional categories were incorporated into the model to fit NC product category requirements, namely automotive waste, aerospace and aviation waste, medical waste, and paper waste. The fate of these waste categories, either ending up in WIP, landfilling, WWTP, reprocessing, or export, was determined following the methodology in Adam and Nowack, ${ }^{44}$ using waste collection and treatment data from reports and the Eurostat database. ${ }^{45}$ As there is no NCspecific sewage treatment plant (STP) removal efficiency study available to our knowledge, we used the data from Sun et $a{ }^{33}{ }^{33}$ for CNT, where the results from STP removal studies were treated with various DoBs, depending on the size of the studies (full-scale STP, pilot STP, or laboratory experiments).

Modelling of the stocks and flows of NC through its life cycle allowed us to quantify the flows into the environment. The surface water compartment was used for the risk assessment undertaken in this study, therefore its predicted environmental concentration (PEC) was calculated. To determine the PEC of the surface water compartment required for the risk characterization ratio calculation, the mass of $\mathrm{NC}$ of a specific year within an environmental compartment is divided by the EU volume of that compartment, as described in detail by Sun et $a l .{ }^{33}$ A PEC distribution was developed by dividing each value making up the mass distribution within the surface water compartment by the compartment volume.

\subsection{Hazard assessment}

In order to assess the hazard, a probabilistic species sensitivity distribution (PSSD) was computed. This was done by compiling NC ecotoxicological endpoints from the literature. The same selection criteria as used by Coll et al. and Gottschalk and Nowack ${ }^{27,46}$ were applied, namely only considering in vivo studies looking at effects on survival, growth, and reproduction, exposed to either freshwater, air or soil.

We applied a probabilistic SSD, ${ }^{46}$ useful when working with small datasets as it produces a sensitivity distribution for each species rather than using a single toxicity endpoint. The compiled endpoints were converted into chronic no effect concentrations (NOEC), using two assessment factors. The time assessment factor (AFt) extrapolated acute studies into chronic estimations, and the dose-effect assessment factor (AFe) converted various endpoints (e.g. $\mathrm{LC}_{50}, \mathrm{EC}_{50}$ ) into NOECs. ${ }^{47}$ All these sensitivity distributions together form the species sensitivity distribution for an entire environmental compartment. The 5th percentile of the resulting PSSD was used as predicted no effect concentration (PNEC), as per European Chemicals Agency (ECHA) guidelines. ${ }^{47}$ We ran 10000 iterations, resulting in a PNEC distribution rather than a single value. Further detail regarding the methodology can be found in Coll et al. and Wigger et al. ${ }^{27,48}$

\subsection{Risk assessment}

In order to characterize the environmental risk surrounding NC according to the European Regulation, ${ }^{49}$ both the exposure and hazard needed to be assessed. This was done by determining the predicted environmental concentration (PEC) 
through an exposure assessment, and the predicted no-effect concentration (PNEC) through a hazard assessment. The risk characterization ration (RCR) was then calculated by dividing the PEC by the PNEC:

$$
\text { RCR }=\text { PEC/PNEC }
$$

An RCR greater than 1 indicates that a risk does exist within the environmental compartment under consideration (surface water, soil, etc.), and that further risk management actions are required. An RCR below 1 indicates that there is no risk under the considered study conditions. In light of the probabilistic approach taken in this study, an RCR distribution, rather than a single value, was calculated by dividing all values of the PEC distribution by all values of the PNEC distribution. ${ }^{27,28}$

\section{Results}

\subsection{Exposure assessment}

3.1.1 Production volume over time. Following the methodology presented in Gottschalk et al. ${ }^{34}$ the production volume distribution was computed based on three data sources regarding NC production volumes (Table S1†). Fig. 1 shows the evolution of nanocellulose production. Production grows from an average $1405 \mathrm{t}$ in $2010\left(Q_{15}=84 \mathrm{t}, Q_{85}=2971 \mathrm{t}\right)$, to $1865 \mathrm{t}$ in $2015\left(Q_{15}=181 \mathrm{t}, Q_{85}=5212 \mathrm{t}\right)$, and up to $30603 \mathrm{t}$ in $2025\left(Q_{15}=1431 \mathrm{t}, Q_{85}=64157 \mathrm{t}\right)$. Due to the optimistic growth forecasts of NC production, there is a steep increase in volume between 2015 and 2025. The decrease in uncertainty in 2015 visible from the grey lines in Fig. 1 is due to it being the reference year, i.e. the year from which the production volume data used in the model is based. The further away a year is from the base year, the larger the relative uncertainty becomes in the model.

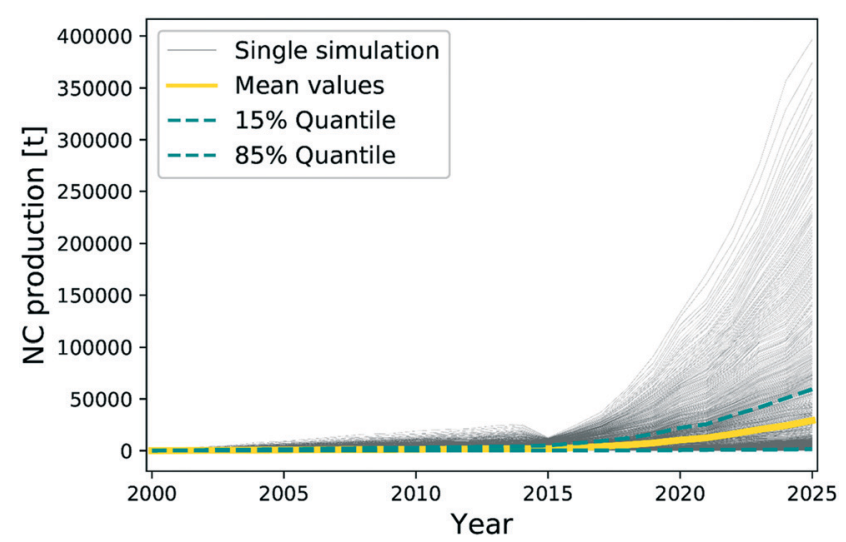

Fig. 1 Evolution of nanocellulose production between 2000 and 2025 in Europe. Each grey line represents a single simulation, the yellow line the mean of these simulations, and the two blue lines the $15 \%$ and $85 \%$ quantiles. The uncertainty for years prior to 2015 is presented because no historical data on the true development of nanocellulose are available.
3.1.2 Product distribution. In our reference year 2015, the 'R\&D' category represents approximately three quarters of the share of produced NC, used in pilot scale plants or for research purposes (Fig. 2). This share was inferred from European production capacity of pilot versus commercial scale plants ${ }^{9}$ and discussed with experts, as we were unable to find more precise information in the literature or market reports. The remaining $25 \%$ is attributed to various applications identified in the literature, the largest shares being 'paper and board', followed by 'packaging' and 'filtration and separation'.

We assumed that $100 \%$ of nanocellulose produced in 2000 went into R\&D, and that this share decreases linearly to 2025, using the 2015 shares mentioned above as a reference. Fig. 2 shows the mean share of each product category. The minimal, mean and maximal values used for the distributions can be found in Table $\mathbf{S} 2 . \dagger$

For ease of reading, we merged the categories representing under 3\% of applications in 2025 into an 'other' category in Fig. 2. These are 'aerogels', 'rheology modifiers', 'printed and flexible electronics', 'rubber and tire additives', and 'colorants'. However, each of these categories was treated independently in the model, with specific lifespans, release dynamics and EoL fate. The 'medical and healthcare' category, within 'other', was further subcategorized in the model, as the types of applications identified in the literature were quite varied, with different EoL and waste management implications. It is composed of 'drug delivery', 'medical implants', 'tissue engineering', 'wound dressings', and 'lateral flow immunoassay labels'. The share of the 'medical and healthcare' category was evenly distributed between these five subcategories, as no detailed information regarding each application was found.

3.1.3 Release dynamics. The flows from 'PMC' to the various stocks and sinks is dependent on the product distribution, as different products will have different lifespans, release characteristics, and EoL fate (Table 1). Each application of the product distribution was characterized in terms of use and release dynamics. For a given application, a certain share of NC contained in the product will be released during its use phase, over a certain amount of time depending on the

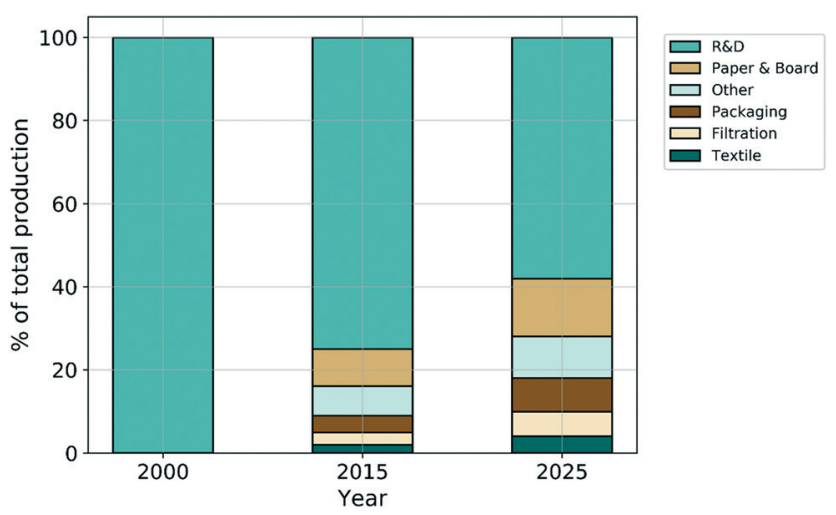

Fig. 2 Allocation of nanocellulose to product categories in 2000, 2015, and 2025. 


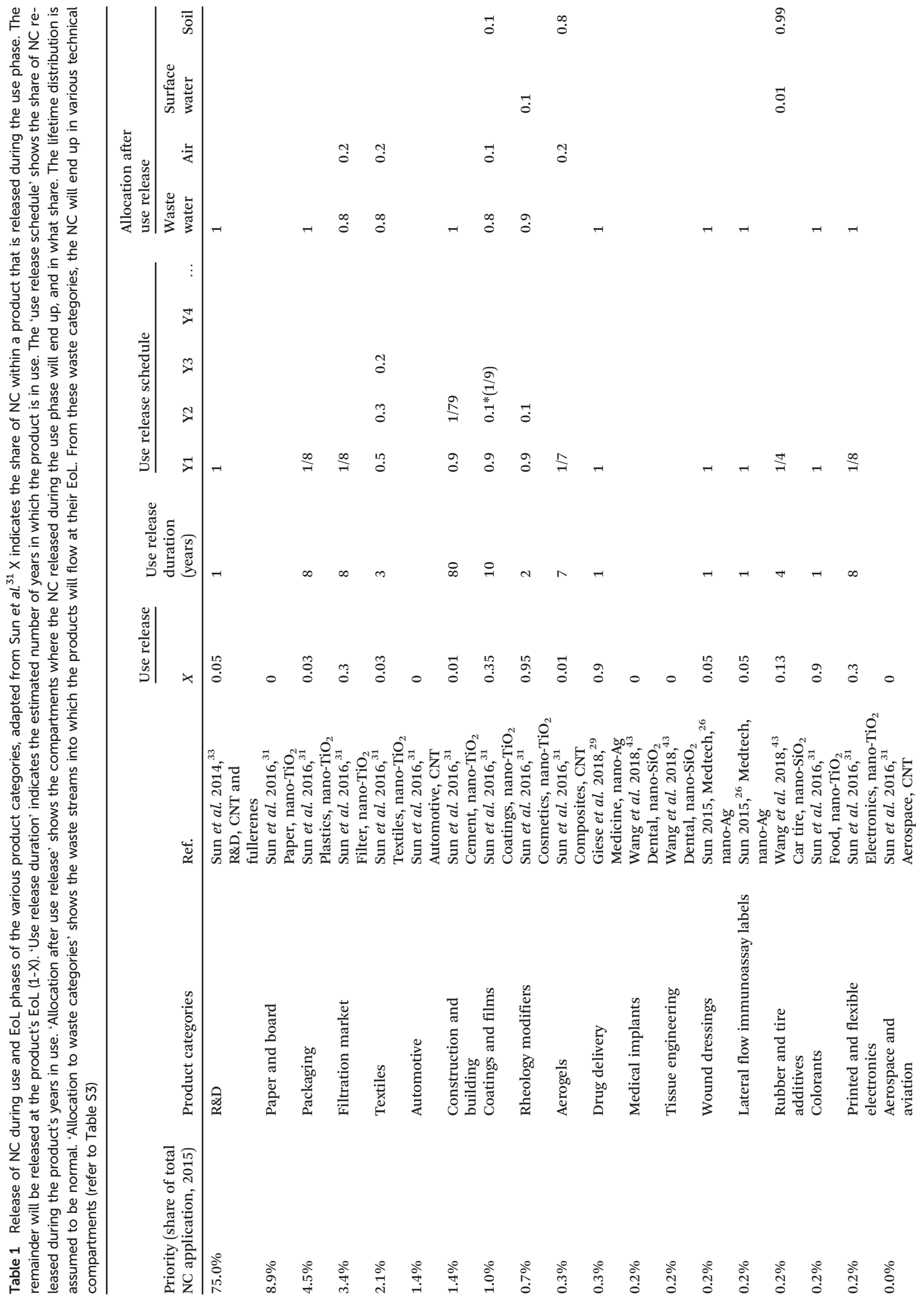




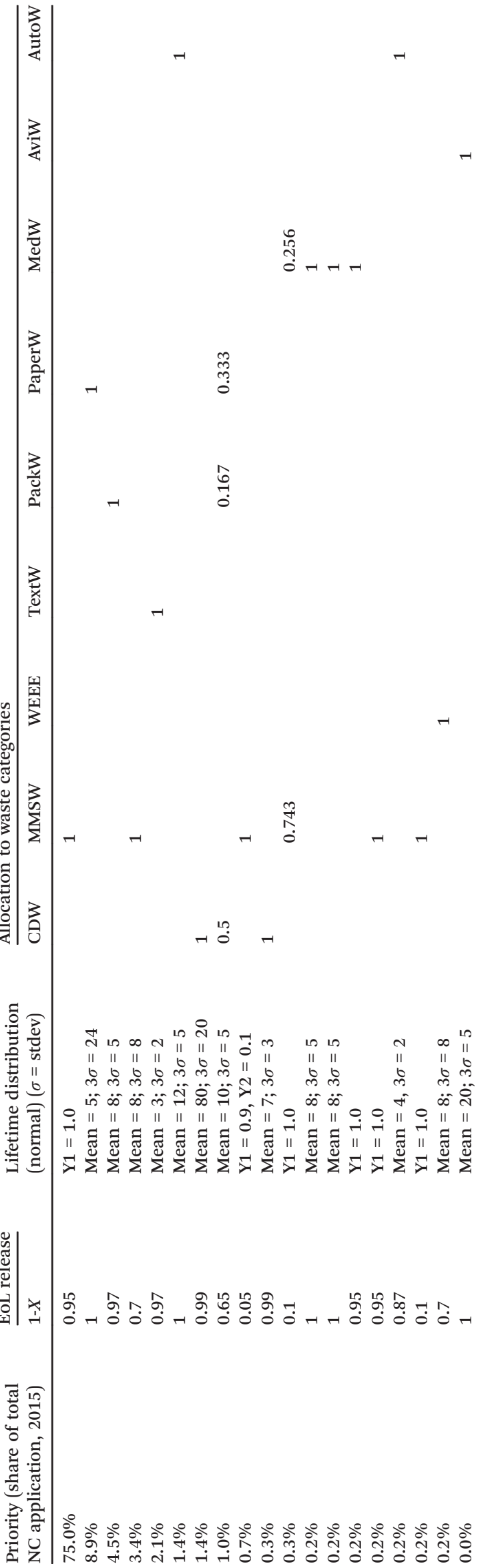

product's lifetime and characteristic. This NC will end up in various environmental compartments. Data relating to the release of NC from various applications remains scarce. Therefore, in our model we used data from studies that looked at similar applications but containing other nanomaterials, as can be seen in the "reference-column" of Table 1. It should be noted that this creates considerable uncertainty surrounding these numbers, and details about the assumptions made can be found in the ESI. $\dagger$ The NC that was not released during the use phase will be released during the product's EoL, again over a certain amount of time depending on the product's characteristics, which will also define in which waste category the product and NC will end up. The fate of the various waste categories is based on European waste management data, and from there will enter various technical and/or environmental compartments (Table S3†).

For example, all NC from the 'paper and board' product category is released at the products' EoL, with there being no use-release for this category. Its EoL-release is split between $82 \%$ going to 'reprocessing' (mean percentage of distribution), and the remaining $18 \%$ going to mixed municipal solid waste (MMSW), ending up in 'landfilling' and 'WIP'. Another example is the 'textile' application, where $3 \%$ of the materials is released during the 3 year average use-phase of the product. Of this $3 \%, 50 \%$ is released into 'wastewater' and 'air' in the first year, $30 \%$ in the second and $20 \%$ during the third. The remaining $97 \%$ is released at the products' EoL, with close to a third being sorted, and the remained going to MMSW ('landfill' and 'WIP').

3.1.4 Flows, stocks and sinks. Nanocellulose release in 2015 was calculated by considering the historical input from 2000 and considering product-specific direct and delayed releases. The same was done for 2025 , by considering future NC demand volume estimates. The distribution of NC inflows between the various technical and environmental stocks and sinks in 2015 and 2025 can be seen in Fig. 3. Although all compartments see an increase in volume of inflows between the two years, inflows to the 'elimination', and 'landfill' compartments, already the largest in 2015 , increase most markedly by 2025 .

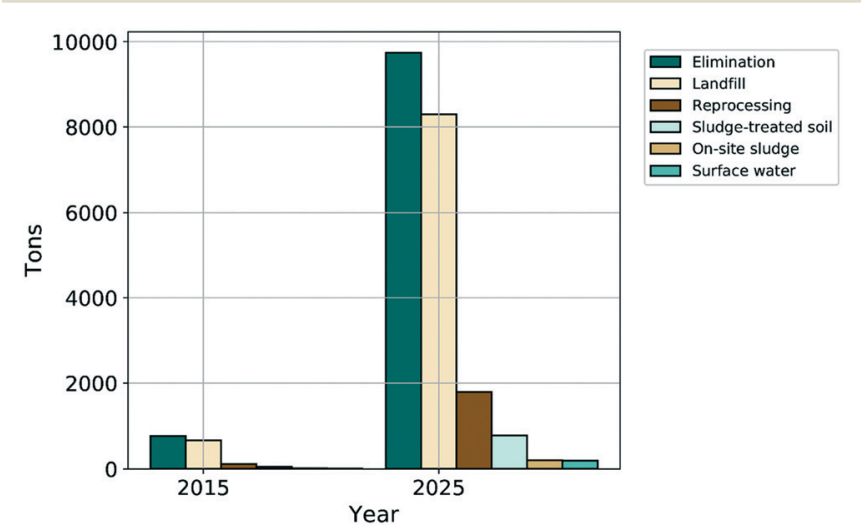

Fig. 3 Inflow of nanocellulose to various stocks and sinks in 2015 and 2025 in Europe. 'ST soil' represents sludge-treated soil. 
The mass flows of NC in 2015 and 2021 and the links between the various compartments are represented in Fig. 4. The model input flow is the production volume, in tons per year. The largest share of this flow is directly released to the various technical compartments. Just under a third of the inflow to 'production', 'manufacturing', and 'consumption' (PMC) enters the 'in-use stock', which will be released during later time-periods, as certain considered products have lifetimes of over one year. In both 2015 and 2025, the volume of NC in the 'in-use' stock is almost equal to the yearly volume being produced. Detailed flow results can be found in Table S4. $\dagger^{\dagger}$

The 'landfill' compartment represents by far the largest sink within the system boundary, having accumulated over $46000 \mathrm{t}$ by 2025. Most NC entering 'WIP' will end up in the 'elimination', which has accumulated over 53000 t by 2025 . Similarly to the 'in-use stock', flows to the three technical sink compartments ('landfill', 'reprocessing' and 'on-site treatment') show high accumulation rates of NC compared to yearly inflows. This is also true for the flows into the environmental sink compartments ('soils', 'surface water' and 'air'), although the flows are smaller than those to technical compartments. This temporal consideration of past production and release dynamics greatly affects the volumes of $\mathrm{NC}$ within a compartment.

Certain applications are likely to release NC into wastewater during their use period, for example 'textiles', 'rheology modifiers', or 'construction and building' materials, as well as the aforementioned ' $R \& D$ ' category. The largest share of NC entering the 'wastewater' compartment will flow into the 'sewage treatment plant' and end up in 'soils', 'WIP' or 'landfills'. The remainder either goes into the 'on-site treatment' compartment, or exits the system to the 'subsurface water' or 'surface water' sinks. Lastly, materials leaving the system through 'export' are shares of WEEE, automotive waste, and textile waste that will be further processed outside of the system boundary.

3.1.5 Dynamics. The evolution of the 'in-use' stock, and 'elimination', 'soil' (both ST soil and NU soil), 'surface water' sinks can be seen in Fig. 5. The 'soil' sink, specifically ST soil, is the environmental compartment that accumulates the largest amount of $\mathrm{NC}$, and although the volumes released to the aquatic environment are relatively low, they are relevant to the risk assessment performed in this study. Many potential applications have a lifetime longer than a single year, explaining the strong, steady growth of the 'in-use' stock, as the $R \& D$ share slowly relatively decreases with time and more applications enter the market. The growth of NC in the 'elimination' compartment, representing the NC that has been combusted in the WIP, is also tied to this increasing commercialization and to many applications being collected in MMSW, ending up in 'WIP' and 'landfill'.

\subsection{Hazard assessment}

Five ecotoxicology studies meeting our probabilistic species sensitivity distribution (PSSD) criteria were identified through a literature review. These covered 8 species and 76 endpoints, all representing the surface water compartment. The available data restricted us to only develop a PSSD for the surface water compartment, with there being insufficient endpoints for soil. The extensive aquatic toxicity study by Kovacs et al. ${ }^{50}$ was the source of 53 of these endpoints. Complete information regarding studies, species, concentrations, and endpoints can be found in Table S5.†

The PSSD was build by combining the sensitivity distributions of the eight species (Fig. 6). Taking the 5th percentile of the PSSD allowed us to calculate the predicted no effect concentration (PNEC) probability density distribution (Fig. S1†), made up of 10000 values from the 10000 PSSD iterations. The mean value of the PNEC distribution is $7.69 \mathrm{mg} \mathrm{l}^{-1}$, the mode $2.11 \mathrm{mg} \mathrm{l}^{-1}, Q_{15} 1.86 \mathrm{mg} \mathrm{l}^{-1}$, and $Q_{85} 14.08 \mathrm{mg} \mathrm{l}^{-1}$.

\subsection{Risk characterization}

The PEC distributions for 2015 and 2025 were computed based on the mass of NC in the surface water in those years, resulting from the Dynamic Probabilistic Material Flow Analysis (DPMFA). To determine the concentration of NC within the surface water compartment, the mass of $\mathrm{NC}$ in the surface water compartment was divided by the volume of the compartment. As the mass in the surface water compartment is a probability density function, each value was divided by the compartment volume in order to obtain a predicted environmental concentration (PEC) distribution. In Fig. 7, the PEC and predicted no effect concentration (PNEC) distribution are compared on a logarithmic scale, for both 2015 and 2025. No overlap is visible for either years, implying that there is no risk considering the conditions of this study. We can however see that the PEC distribution in 2025 is slightly further to the right than in 2015, indicating an increase in concentration between these two years.

The risk characterization ratio (RCR) distributions for 2015 and 2025 were computed by dividing all the values of the PEC distribution by all the values of the PNEC distribution (Fig. S2a and S2b $\dagger$ ). In order to determine single RCR values, the mean, mode, median, $Q_{15}$ and $Q_{85}$ values were calculated (Table 2).

\section{Discussion}

This study aimed to quantitatively assess the environmental risk of nanocellulose (NC) within Europe using a dynamic and probabilistic modeling approach. Overall, the results show a low risk surrounding this material, considering both current production volumes as well as future growth projections. Comparing the risk characterization ration (RCR) value for NC with those of other ENMs from past studies allows for a better contextualization of its risk within the nanomaterial domain. The study by Coll et $a .^{27}$ that looked at the risk of five nanomaterials, showed RCR mode values in the freshwater compartment ranging from 0.03 for nano- $\mathrm{TiO}_{2}$, to $<0.01$ for CNT and fullerenes. The RCR of NC is therefore similar to those of these two carbon-based ENMs, nearing zero. Even 

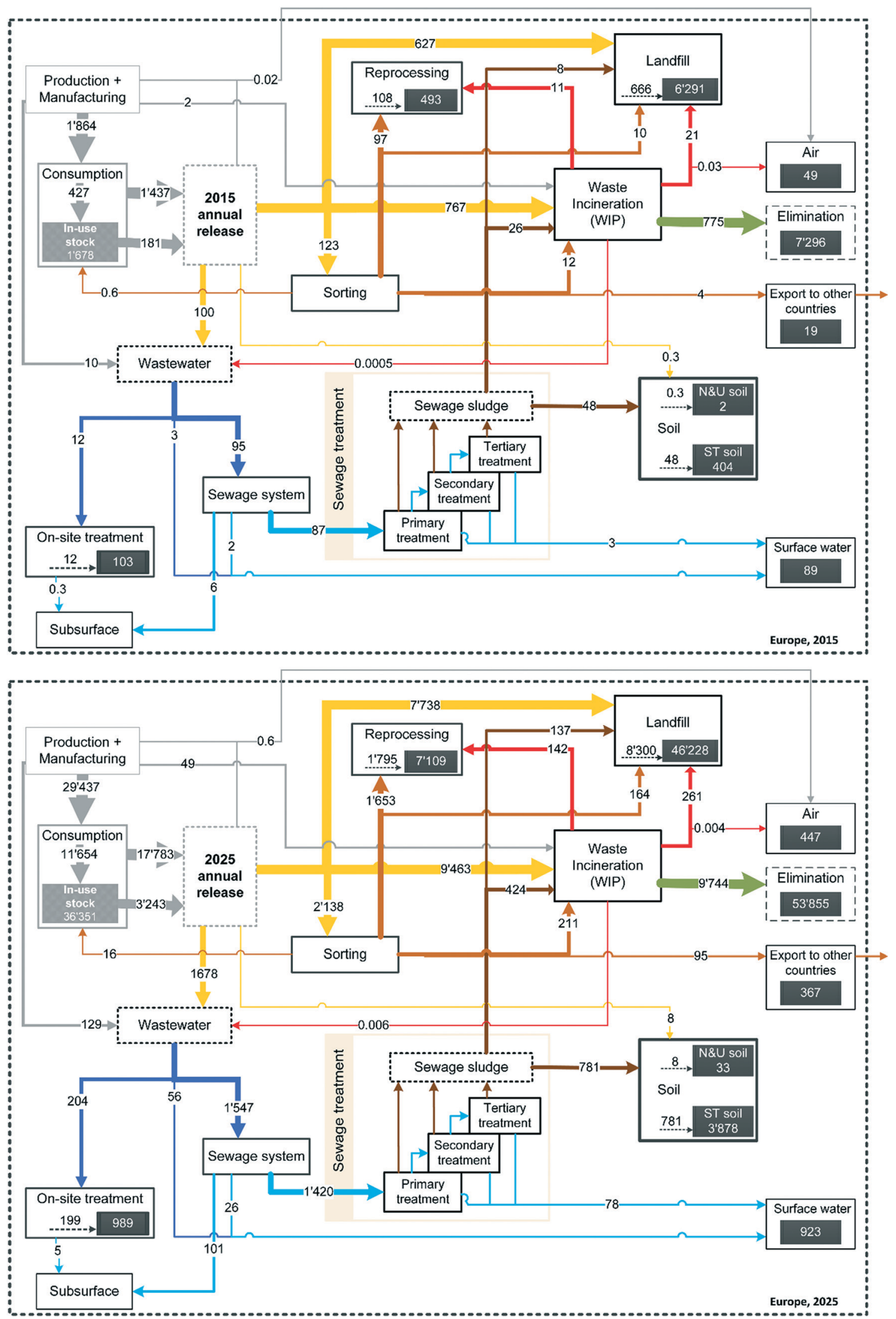

Fig. 4 Results of material flows of nanocellulose in 2015 (top) and 2025 (bottom) in tons in Europe. Values are means of the probabilistic distributions. Technical and environmental compartments are represented as boxes, and flows between these compartments as arrows, with arrow thickness varying based on volumes of the flows. The black boxes within compartments represent stocks, i.e. the amount of NC having accumulated over time. 

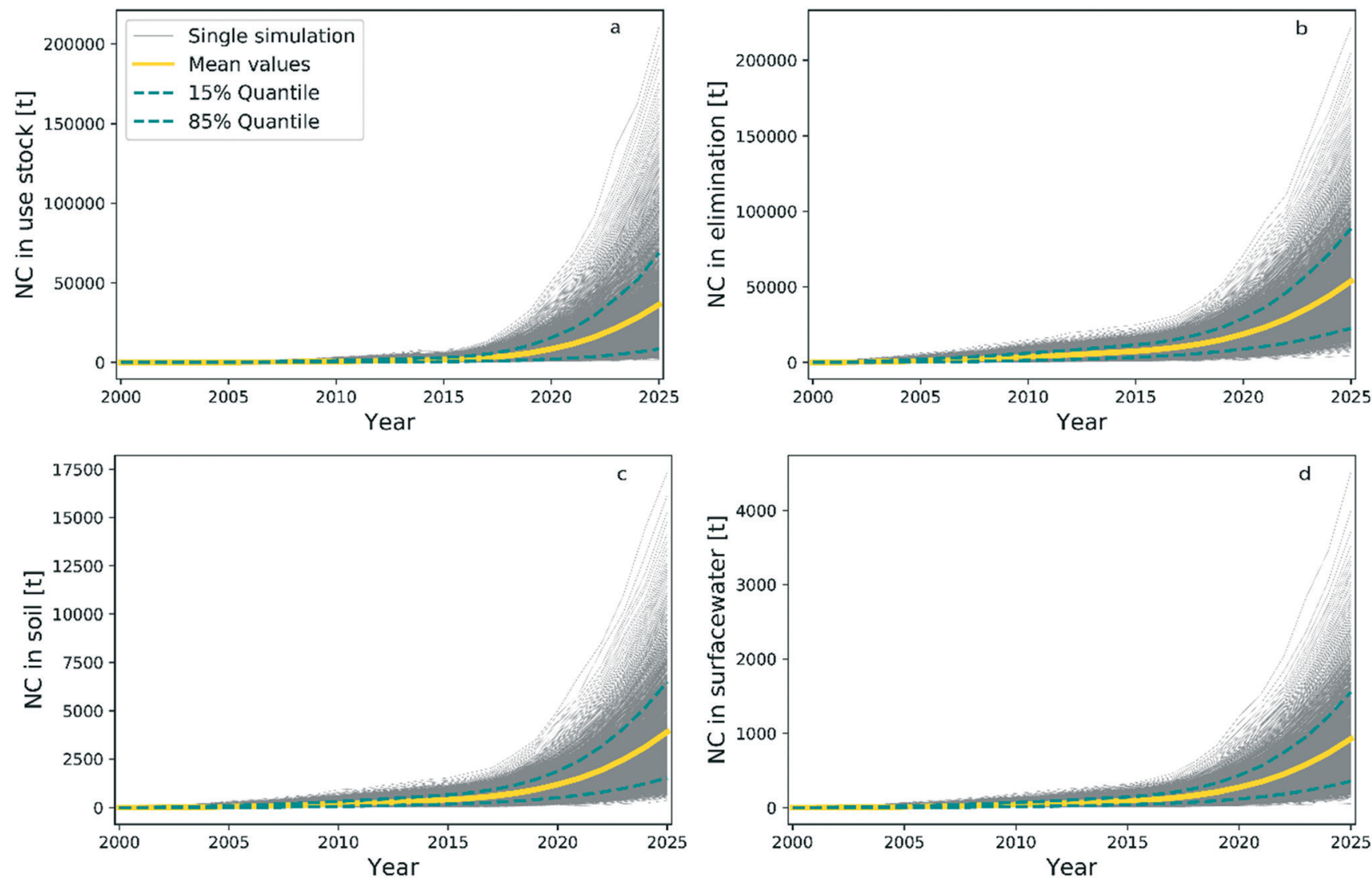

Fig. 5 Evolution of (a) 'in-use', (b) 'elimination', (c) 'soil' (ST soil and NU soil), and (d) 'surface water' compartments in Europe. Different $y$-axis ranges were used to better represent the growth of each compartment.

when considering increased accumulation and production of NC over time, the RCR value for 2025 remains well below one, indicating that toxicity values are greater than the PEC in the freshwater compartment. These results were expected due to the nanomaterial's bulk form, cellulose, being nontoxic, and past toxicological studies having largely shown low toxicity of the material (e.g. ref. 51-53). However, the ecotoxicological literature surrounding NC remains scarce, and a number of studies put forward the existence of knowledge and data gaps. ${ }^{17,20}$

Current and future production volumes of NC remain highly uncertain. Although using a probabilistic approach does allow to account for this high uncertainty, having more sources would increase the robustness of the model. The three sources used as input data for our model varied substantially, and the lack of current and past production data means that any forecast needs to be treated with caution. ${ }^{12}$ On the other hand, the high volumes used as input in this model allow to represent a 'worst case scenario', with high production volumes leading to high concentrations in technical and environmental compartments, and therefore also a 'worst case' RCR. As our model is based on data from 2015, a qualitative comparison of 2019 predictions and its current status show the rate of commercialization is likely overestimated in the production data sources used. Although information regarding the current status of nanocellulose- based products on the market was difficult to come by, informal interviews with experts in the field corroborated out assumption about overestimated 2019 volumes, as many applications are still at pilot scale, and the predicted growth in production not yet having been observed.

In 2015, three quarters of produced $\mathrm{NC}$ was allocated to $\mathrm{R} \& \mathrm{D}$, as many applications are only expected to reach commercialization in the upcoming years. This strongly influences the flows of the material within the system, as the NC in the R\&D category was treated as having a lifespan of one year, with 95\% of it being released at its EoL into mixed municipal solid waste (MMSW), and $5 \%$ being released into wastewater during its use phase. As applications reach commercialization over the years, thereby reducing the share of R\&D, the stocks and flows will likely significantly change. As more products reach commercialization, the flows into sorting and reprocessing will likely grow, as many of the more prominent product categories have high recycling rates, for example paper and board, packaging, and textiles. Our model currently treats reprocessing as a sink. However, a next step would be to make this more realistic by detailing the fate of NC entering this compartment, and identifying in which environmental or technical compartments it may end up.

We were only able to compute a PSSD for the freshwater compartment due to missing data availability for soils and sediments. The choice of Kovacs et al. ${ }^{50}$ to focus on aquatic 


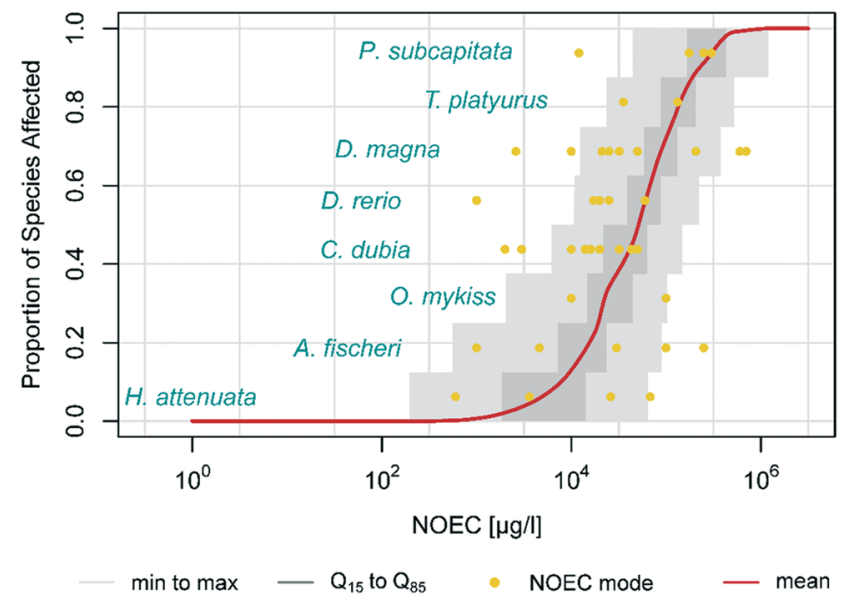

Fig. 6 Probabilistic species sensitivity distribution (PSSD) of nanocellulose in freshwater, based on NOEC values. Yellow points represent single endpoint concentration values.

species for an in-depth ecotoxicity study of NC was justified by the wastewater stream having the highest potential for accidental release, with releases to soils and air considered improbable on a broad scale. However, the results from the DPMFA in this study show significant flows of NC to both natural and urban soils and sludge treated soils, and particularly important accumulations in the latter via wastewater treatment plants. There is also release directly from the use phase of products, due to applications such as rubber and tire additives and rheology modifiers, which are likely to grow in the future. The study by Vikman et al. ${ }^{54}$ found cellulose nanofibers (CNF) based packaging products to be biodegradable and compostable, and the ecotoxicological test performed in a compost environment did not show toxicity. However, more ecotoxicity tests looking at species in the soil compartment would be useful to develop a PSSD and quantitatively assess the risk within this compartment. Additional studies looking at the freshwater compartment would also help strengthen our results. According to European require-

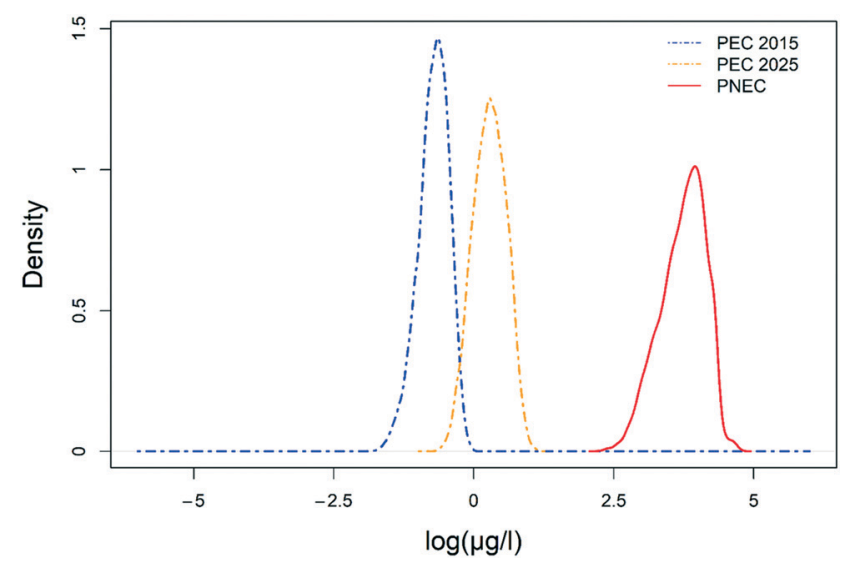

Fig. 7 Comparison of the 2015 and 2025 predicted environmental concentration (PEC) distributions and the predicted no-effect concentration (PNEC) distribution. An overlap of PEC and PNEC curves would signify that a risk is present.
Table 2 Risk characterization ratio of nanocellulose in the surface water compartment in 2015 and 2025

\begin{tabular}{llllll}
\hline & Mean & Mode & Median & $Q_{15}$ & $Q_{85}$ \\
\hline 2015 & $6.9 \times 10^{-5}$ & $2.0 \times 10^{-5}$ & $3.4 \times 10^{-5}$ & $1.1 \times 10^{-5}$ & $1.2 \times 10^{-4}$ \\
2025 & $7.1 \times 10^{-4}$ & $2.6 \times 10^{-4}$ & $3.4 \times 10^{-4}$ & $1.0 \times 10^{-4}$ & $1.2 \times 10^{-3}$
\end{tabular}

ments, ${ }^{47}$ a minimum of ten species from eight taxonomic group are necessary to create a robust SSD. The PSSD in this study was based on eight species covering seven taxonomic groups. Although still allowing to produce useful results, these could be reinforced with additional aquatic ecotoxicological NC endpoints.

The PEC values reported in our model do not include any environmental fate processes such as degradation or agglomeration and sedimentation. We are therefore not providing real environmental concentrations but "release concentrations", quantifying the total amount of NC that ends up in an environmental compartment. By considering environmental fate processes, environmental fate models for nanomaterials such as SimpleBox $4 \mathrm{Nano}^{55}$ or NanoFate ${ }^{56}$ are able to predict distribution and concentrations in different environmental compartments. ${ }^{57}$ These fate models use the environmental release data provided by material flow models such as the one used in our current work. The PEC values provided by our model constitute a worst-case assessment by not considering potential degradation or agglomeration of particles. Within a prospective assessment, such an approach is justified, especially considering the fact that the exposure concentrations are many orders of magnitude smaller than the PNEC. Therefore reducing the PEC will even further decrease the risk characterization ratio.

A validation of the modelled released amounts or PEC values by measurements is not possible at the moment. This issue is not specific to NC but hampers all nanomaterial exposure assessments. Nowack et $a .^{21}$ have discussed in detail that the results currently provided by analytical methods are not yet specific to engineered nanomaterials or are just not sensitive enough to reach realistic environmental concentrations. The available measurements cannot currently be used to validate the results of modeling studies, but they can provide orthogonal information to get a complete understanding of the presence of natural and engineered nanoparticles in the environment.

\section{Conclusion}

Considering the interest that $\mathrm{NC}$ is receiving from research and industry, as well as the number of novel applications rapidly being developed, it is crucial that in-depth risk analyses are performed to ensure that all safety aspects have been considered before commercialization. Despite the significant uncertainties surrounding the NC data used in our model, this study allows for a first prospective assessment of NC, at a regional scale with concentrations averaged across the territory. A next step would be to assess local concentrations, for 
a more detailed view of potential hotspots and variations within Europe, although the current scarcity of information regarding NC production would make this challenging. Nevertheless, results from this first quantitative risk assessment give an optimistic outlook for the future of this material.

\section{Conflicts of interest}

There are no conflicts to declare.

\section{Acknowledgements}

We would like to thank Dr. Véronique Adam for her helpful comments on an earlier draft. Thank you to the GREENSENSE consortium and Thomas Geiger for useful discussions. This project has received funding from the European Union's Horizon 2020 research and innovation programme under grant agreement No. 761000 GREENSENSE.

\section{References}

1 Y. Xue, Z. Mou and H. Xiao, Nanocellulose as a sustainable biomass material: Structure, properties, present status and future prospects in biomedical applications, Nanoscale, 2017, 9(39), 14758-14781.

2 J. A. Shatkin, T. H. Wegner, E. Bilek and J. Cowie, Market projections of cellulose nanomaterial-enabled products Part 1: Applications, Tappi J., 2014, 13(5), 9-16.

3 P. Scarfato, L. Di Maio and L. Incarnato, Recent advances and migration issues in biodegradable polymers from renewable sources for food packaging, J. Appl. Polym. Sci., 2015, 132(48), 132.

4 H. Kargarzadeh, J. Huang, N. Lin, I. Ahmad, M. Mariano and A. Dufresne, et al., Progress in Polymer Science Recent developments in nanocellulose-based biodegradable polymers , thermoplastic polymers, and porous nanocomposites, Prog. Polym. Sci., 2018, 87, 197-227.

5 Y. Su, B. Yang, J. Liu, B. Sun, C. Cao and X. Zou, et al., Prospects for replacement of some plastics in packaging with lignocellulose materials: A brief review, BioResources, 2018, 13(2), 4550-4576.

6 P. Gatenholm and D. Klemm, Bacterial nanocellulose as a renewable material for biomedical applications, MRS Bull., 2010, 35(3), 208-213.

7 D. Klemm, E. D. Cranston, D. Fischer, M. Gama, S. A. Kedzior and D. Kralisch, et al., Nanocellulose as a natural source for groundbreaking applications in materials science: Today's state, Mater. Today, 2018, 21(7), 720-748.

8 H. Charreau, M. Foresti and A. Vazquez, Nanocellulose Patents Trends: A Comprehensive Review on Patents on Cellulose Nanocrystals, Microfibrillated and Bacterial Cellulose, Recent Pat. Nanotechnol., 2012, 7(1), 56-80.

9 Future Markets Inc., The Global Market for Nanocellulose, Edinburgh, 2015.

10 R. J. Moon, G. T. Schueneman and J. Simonsen, Overview of Cellulose Nanomaterials, Their Capabilities and Applications, JOM, 2016, 68(9), 2383-2394.
11 K. Nelson, Agenda 2020 Cellulose Nanomaterials Research Roadmap, Washington, DC, 2016.

12 J. Miller, Nanocellulose: Producers, Products, and Applications, Peachtree Corners, 2017.

13 S. J. Klaine, A. A. Koelmans, N. Horne, S. Carley, R. D. Handy and L. Kapustka, et al., Paradigms to assess the environmental impact of manufactured nanomaterials, Environ. Toxicol. Chem., 2012, 31(1), 3-14.

14 J. L. Gardea-Torresdey, C. M. Rico and J. C. White, Trophic transfer, transformation, and impact of engineered nanomaterials in terrestrial environments, Environ. Sci. Technol., 2014, 48(5), 2526-2540.

15 C. Endes, S. Camarero-Espinosa, S. Mueller, E. J. Foster, A. Petri-Fink and B. Rothen-Rutishauser, et al., A critical review of the current knowledge regarding the biological impact of nanocellulose, J. Nanobiotechnol., 2016, 14(1), 1-14.

16 A. Kermanizadeh, I. Gosens, L. MacCalman, H. Johnston, P. H. Danielsen and N. R. Jacobsen, et al., A Multilaboratory Toxicological Assessment of a Panel of 10 Engineered Nanomaterials to Human Health - ENPRA Project - The Highlights, Limitations, and Current and Future Challenges, J. Toxicol. Environ. Health, Part B, 2016, 19(1), 1-28.

17 M. Roman, Toxicity of Cellulose Nanocrystals: A Review, Ind. Biotechnol., 2015, 11(1), 25-33.

18 E. Lie, E. Ålander and T. Lindström, Possible toxicological effects of nanocellulose - An updated literature study, No 2, Stockholm, 2017.

19 A. B. Seabra, J. S. Bernardes, W. J. Fávaro, A. J. Paula and N. Durán, Cellulose nanocrystals as carriers in medicine and their toxicities: A review, Carbohydr. Polym., 2018, 181, 514-527.

20 J. A. Shatkin and B. Kim, Cellulose nanomaterials: life cycle risk assessment, and environmental health and safety roadmap, Environ. Sci.: Nano, 2015, 2(5), 477-499.

21 B. Nowack, M. Baalousha, N. Bornhöft, Q. Chaudhry, J. Lead and D. M. Mitrano, et al., Progress towards the validation of modeled environmental concentrations of engineered nanomaterials by analytical measurements, Environ. Sci.: Nano, 2015, 2, 421-428.

22 F. Gottschalk, T. Sondere, R. Schols and B. Nowack, Modeled Environmental Concentrations of Engineered Nanomaterials for Different Regions, Environ. Sci. Technol., 2009, 43(24), 9216-9222.

23 A. A. Keller and A. Lazareva, Predicted Releases of Engineered Nanomaterials: From Global to Regional to Local, Environ. Sci. Technol. Lett., 2013, 1(1), 65-70.

24 A. A. Keller, S. McFerran, A. Lazareva and S. Suh, Global life cycle releases of engineered nanomaterials, J. Nanopart. Res., 2013, 15(6), 15.

25 F. Gottschalk, E. Kost and B. Nowack, Engineered nanomaterials in water and soils: A risk quantification based on probabilistic exposure and effect modeling, Environ. Toxicol. Chem., 2013, 32(6), 1278-1287.

26 I. Mahapatra, T. Y. Sun, J. R. A. Clark, P. J. Dobson, K. Hungerbuehler and R. Owen, et al., Probabilistic modelling of prospective environmental concentrations of gold 
nanoparticles from medical applications as a basis for risk assessment, J. Nanobiotechnol., 2015, 13(1), 1-14.

27 C. Coll, D. Notter, F. Gottschalk, T. Sun, C. Som and B. Nowack, Probabilistic environmental risk assessment of five nanomaterials (nano-TiO2, nano-Ag, nano-ZnO, CNT, and fullerenes), Nanotoxicology, 2016, 10(4), 436-444.

28 Y. Wang, A. Kalinina, T. Sun and B. Nowack, Probabilistic modeling of the flows and environmental risks of nanosilica, Sci. Total Environ., 2016, 545-546, 67-76.

29 B. Giese, F. Klaessig, B. Park, R. Kaegi, M. Steinfeldt and H. Wigger, et al., Risks, Release and Concentrations of Engineered Nanomaterial in the Environment, Sci. Rep., 2018, 8(1), 1-18.

30 N. A. Bornhöft, T. Y. Sun, L. M. Hilty and B. Nowack, A dynamic probabilistic material flow modeling method, Environ. Model. Softw., 2016, 76, 69-80.

31 T. Y. Sun, N. A. Bornhöft, K. Hungerbühler and B. Nowack, Dynamic Probabilistic Modeling of Environmental Emissions of Engineered Nanomaterials, Environ. Sci. Technol., 2016, 50(9), 4701-4711.

32 R. Song, Y. Qin, S. Suh and A. A. Keller, Dynamic Model for the Stocks and Release Flows of Engineered Nanomaterials, Environ. Sci. Technol., 2017, 51(21), 12424-12433.

33 T. Y. Sun, F. Gottschalk, K. Hungerbühler and B. Nowack, Comprehensive probabilistic modelling of environmental emissions of engineered nanomaterials, Environ. Pollut., 2014, 185, 69-76.

34 F. Gottschalk, R. W. Scholz and B. Nowack, Probabilistic material flow modeling for assessing the environmental exposure to compounds: Methodology and an application to engineered nano-TiO2particles, Environ. Model. Softw., 2010, 25(3), 320-332.

35 A. D. Martin, K. M. Quinn and J. H. Park, MCMCpack: Markov Chain Monte Carlo in R, J. Stat. Softw., 2011, 1(9), 14.

36 J. Miller, Nanocellulose challenges and opportunities, Peachtree Corners, 2017.

37 E. Inshakova and O. Inshakov, World market for nanomaterials: structure and trends, MATEC Web Conf., 2017, 129.

38 J. Cowie, E. Bilek, T. H. Wegner and J. A. Shatkin, Market projections of cellulose nanomaterial-enabled products - Part 2: Volume estimates, Tappi J., 2014, 13(6), 57-69.

39 D. H. Milanez, R. M. Amaral do, L. I. L. Faria de and J. A. R. Gregolin, Assessing nanocellulose developments using science and technology indicators, J. Mater. Res., 2013, 16(3), 635-641.

40 B. Nowack, R. M. David, H. Fissan, H. Morris, J. A. Shatkin and M. Stintz, et al., Potential release scenarios for carbon nanotubes used in composites, Environ. Int., 2013, 59, 1-11.

41 W. Wohlleben and N. Neubauer, Quantitative rates of release from weathered nanocomposites are determined across 5 orders of magnitude by the matrix, modulated by the embedded nanomaterial, NanoImpact, 2016, 1, 39-45.

42 F. von der Kammer, P. L. Ferguson, P. A. Holden, A. Masion, K. R. Rogers and S. J. Klaine, et al. Analysis of engineered nanomaterials in complex matrices (environment and biota): General considerations and conceptual case studies, Environ. Toxicol. Chem., 2012, 31(1), 32-49.
43 Y. Wang and B. Nowack, Dynamic probabilistic material flow analysis of nano-SiO2, nano iron oxides, nano-CeO2, nano-Al2O3, and quantum dots in seven European regions, Environ. Pollut., 2018, 235, 589-601.

44 V. Adam and B. Nowack, European country-specific probabilistic assessment of nanomaterial flows towards landfilling, incineration and recycling, Environ. Sci.: Nano, 2017, 4(10), 1961-1973.

45 Eurostat. Waste database [Internet]. Available from: https:// ec.europa.eu/eurostat/web/environment/waste/database.

46 F. Gottschalk and B. Nowack, A probabilistic method for species sensitivity distributions taking into account the inherent uncertainty and variability of effects to estimate environmental risk, Integr. Environ. Assess. Manage., 2013, 9(1), 79-86.

47 European Chemicals Agency, Guidance on information requirements and chemical safety assessment Chapter $R$. 10 : Characterisation of dose concentration response for environment. Reproduction, Helsinki, 2008.

48 H. Wigger, D. Kawecki, B. Nowack and V. Adam, Systematic consideration of parameter uncertainty and variability in probabilistic species sensitivity distributions, Integr. Environ. Assess. Manage., under review.

49 European Chemicals Agency, Guidance on Information Requirements and Chemical Safety Assessment. Part E: Risk Characterisation, vol. 2.1, European Chemicals Agency, Helsinki, 2016.

50 T. Kovacs, V. Naish, B. O'Connor, C. Blaise, F. Gagné and L. Hall, et al., An ecotoxicological characterization of nanocrystalline cellulose (NCC), Nanotoxicology, 2010, 4(3), 255-270.

51 B. O'Connor, R. Berry and R. Goguen, Commercialization of Cellulose Nanocrystal (NCC ${ }^{\mathrm{TM}}$ ) Production: A Business Case Focusing on the Importance of Proactive EHS Management, Nanotechnology Environmental Health and Safety: Risks, Regulation, and Management, Elsevier Inc., 2nd edn, 2014, pp. 225-246.

52 K. J. Ong, J. A. Shatkin, K. Nelson, J. D. Ede and T. Retsina, Establishing the safety of novel bio-based cellulose nanomaterials for commercialization, NanoImpact, 2017, 6, 19-29.

53 F. Ghaemi and L. C. Abdullah, Comparative study of cytotoxicity effect between cellulose nanocrystal and cellulose nanofiber, Advances in Nanobiotechnology, 2018, 1, 1-3.

54 M. Vikman, J. Vartiainen, I. Tsitko and P. Korhonen, Biodegradability and Compostability of Nanofibrillar Cellulose- Based Products, J. Polym. Environ., 2015, 206-215.

55 J. A. J. Meesters, A. A. Koelmans, J. T. K. Quik and A. J. Hendriks, Multimedia Modeling of Engineered Nanoparticles with SimpleBox4nano: Model Definition and Evaluation, Environ. Sci. Technol., 2014, 48, 5726-5736.

56 H. H. Liu and Y. Cohen, Multimedia Environmental Distribution of Engineered Nanomaterials, Environ. Sci. Technol., 2014, 48, 3281-3292.

57 A. L. Dale, E. A. Casman, G. V. Lowry, J. R. Lead, E. Viparelli and M. Baalousha, Modeling Nanomaterial Environmental Fate in Aquatic Systems, Environ. Sci. Technol., 2015, 49(5), 2587-2593. 